Article

\title{
Antiviral, Antifungal and Antibacterial Activities of a BODIPY-Based Photosensitizer
}

\author{
Bradley L. Carpenter ${ }^{1}$, Xingci Situ ${ }^{1}$, Frank Scholle ${ }^{2}$, Juergen Bartelmess ${ }^{1}$, Walter W. Weare ${ }^{1}$ \\ and Reza A. Ghiladi ${ }^{1, *}$
}

1 Department of Chemistry, North Carolina State University, Raleigh, NC 27695-8204, USA;

E-Mails: blcarpen@ncsu.edu (B.L.C.); xsitu@ncsu.edu (X.S.); Juergen.Bartelmess@iit.it (J.B.); wwweare@ncsu.edu (W.W.W.)

2 Department of Biological Sciences, North Carolina State University, Raleigh, NC 27695-7614, USA;

E-Mail: fscholl@ncsu.edu

* Author to whom correspondence should be addressed; E-Mail: Reza_Ghiladi@ncsu.edu;

Tel.: +1-919-389-1716; Fax: +1-919-515-5079.

Academic Editor: Peter J. Rutledge

Received: 23 February 2015 / Accepted: 4 June 2015 / Published: 8 June 2015

\begin{abstract}
Antimicrobial photodynamic inactivation (aPDI) employing the BODIPY-based photosensitizer 2,6-diiodo-1,3,5,7-tetramethyl-8-( $N$-methyl-4-pyridyl)-4,4'-difluoroboradiazaindacene (DIMPy-BODIPY) was explored in an in vitro assay against six species of bacteria (eight total strains), three species of yeast, and three viruses as a complementary approach to their current drug-based or non-existent treatments. Our best results achieved a noteworthy 5-6 log unit reduction in CFU at $0.1 \mu \mathrm{M}$ for Staphylococcus aureus (ATCC-2913), methicillin-resistant $S$. aureus (ATCC-44), and vancomycin-resistant Enterococcus faecium (ATCC-2320), a 4-5 log unit reduction for Acinetobacter baumannii ATCC-19606 (0.25 $\mu \mathrm{M})$, multidrug resistant A. baumannii ATCC-1605 $(0.1 \mu \mathrm{M})$, Pseudomonas aeruginosa ATCC-97 $(0.5 \mu \mathrm{M})$, and Klebsiella pneumoniae ATCC-2146 $(1 \mu \mathrm{M})$, and a $3 \log$ unit reduction for Mycobacterium smegmatis $\mathrm{mc}^{2} 155$ (ATCC-700084). A 5 log unit reduction in CFU was observed for Candida albicans ATCC-90028 (1 $\mu \mathrm{M})$ and Cryptococcus neoformans ATCC-64538 $(0.5 \mu \mathrm{M})$, and a $3 \log$ unit reduction was noted for Candida glabrata ATCC-15545 $(1 \mu \mathrm{M})$. Infectivity was reduced by $6 \log$ units in dengue $1(0.1 \mu \mathrm{M})$, by $5 \log$ units $(0.5 \mu \mathrm{M})$ in vesicular stomatitis virus, and by $2 \log$ units $(5 \mu \mathrm{M})$ in human adenovirus-5. Overall, the results demonstrate that DIMPy-BODIPY exhibits antiviral,
\end{abstract}


antibacterial and antifungal photodynamic inactivation at nanomolar concentrations and short illumination times.

Keywords: photodynamic therapy; singlet oxygen; antibacterial; antiviral; antifungal; photobiocidal

\section{Introduction}

Despite the advent of antibiotics and vaccines, infectious diseases remain the leading worldwide cause of mortality and morbidity [1]. Accounting for over $60 \%$ of deaths in the developing world, they are also the third and fourth leading causes of death in Europe and the United States, respectively [1,2]. Efforts to control microbial infections have been hampered by the emergence and proliferation of drug resistant pathogens, necessitating the pursuit of complementary approaches to the current drug-based treatments. Furthermore, a scarcity of effective therapies for many globally important viral infections stresses the need for development of novel approaches for either treatment or prevention of infection. One such option, antimicrobial photodynamic inactivation (aPDI), is currently being explored as a potential therapeutic treatment option for various types of infection, whether bacterial, fungal, viral, or even parasitic in nature [3-7]. aPDI makes use of a photosensitizer (PS) to generate reactive oxygen species (i.e., radicals or singlet oxygen $\left({ }^{1} \mathrm{O}_{2}\right)$ ) upon illumination with light (visible or near infrared). Although challenges with aPDI exist, such as issues of tissue penetration with light and photosensitizer selectivity, as a biocidal agent, ${ }^{1} \mathrm{O}_{2}$ possesses a number of unique properties that make it particularly attractive for antimicrobial applications. These include damaging reactivity with most biomolecules, a short lifetime of $\sim 10^{-6} \mathrm{~s}$ in aqueous environments, and the formation of harmless ground state molecular oxygen if left unreacted [8,9]. More importantly, development of bacterial resistance to aPDI is believed to be unlikely due to the non-specific damage caused by ${ }^{1} \mathrm{O}_{2}$ [10]. Additionally, a number of studies have shown that a photodynamic inactivation strategy is equally effective against both drug-susceptible and drug-resistant bacterial strains, demonstrating the enormous potential of aPDI in combating pathogenic infections $[11,12]$.

While numerous efforts have focused on photosensitizer scaffolds based upon cationic tetrapyrrole-related macrocycles (porphyrins, bacteriochlorins, phthalocyanines) or other conjugated systems [13-16], in particular methylene blue [17-20], very little is known regarding the applicability of boron dipyrromethene (a.k.a. BODIPY)-based compounds as potential photosensitizers for aPDI. BODIPY dyes exhibit a number of properties that are potentially attractive for photodynamic applications, including synthetic tunability of their absorption features, chemical robustness, and excellent hydrolytic stability under physiological conditions [21-25]. Of particular interest for our work are two recent studies: The first, by Caruso et al. [26], investigated two novel cationic and iodinated BODIPYs as photosensitizers, but only against two bacterial model strains, Escherichia coli and Staphylococcus xylosus. The second study, by O'Shea et al. [27], examined the aPDI application of the structurally-related aza-BODIPY photosensitizers against E. coli, S. aureus, and Candida albicans. Outside of these two studies, reports of a BODIPY-based photosensitizer employed in antimicrobial photodynamic inactivation are limited, and to the best of our knowledge no studies have been 
performed against viruses with this class of photosensitizer. Given that the majority of nosocomial infections in the United States are caused by Staphylococcus aureus (15\%), enterococci species (12\%), candida species (11\%), Pseudomonas aeruginosa (8\%), Klebsiella pneumoniae (6\%), enterobacter species (5\%) and Acinetobacter baumannii (3\%) [28,29], the fact that relatively few clinically-relevant pathogenic bacterial and fungal species, and importantly no viruses, have been explored with BODIPY-based systems highlights a lack of understanding of this new class of photosensitizer with pathogens of direct importance to human health and disease. Herein, to further investigations into the feasibility of aPDI for future therapeutic use as a complementary approach to drug-based treatments, we explored the extent of in vitro antimicrobial photodynamic inactivation employing the DIMPy-BODIPY [26,30] photosensitizer (Figure 1) against eight species of bacteria and three species of yeast that together fall within the five classes of antibiotic-resistant pathogens that are emerging as major public health threats: vancomycin-resistant enterococci (VRE), methicillin-resistant Staphylococcus aureus (MRSA), multidrug-resistant mycobacteria, Gram-negative bacteria, and fungi [31]. We also extended our study to include dengue-1 virus, vesicular stomatitis virus (VSV), and human adenovirus-5 as model DNA viruses to explore the potential of antiviral PDI [32-35] using BODIPY-based photosensitizers for non-in vivo surface sterilization or materials applications.

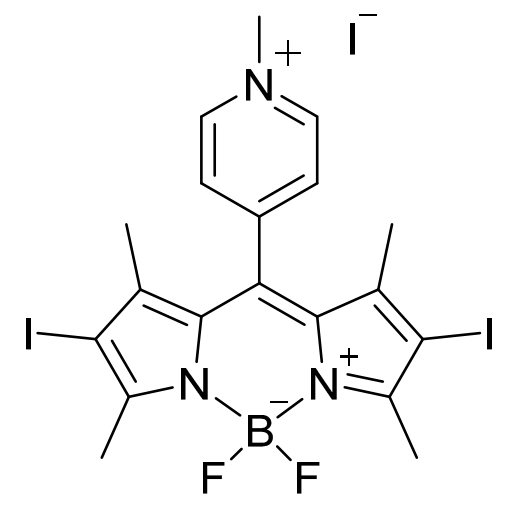

Figure 1. The DIMPy-BODIPY photosensitizer employed in this study.

\section{Results and Discussion}

\subsection{Antiviral Photodynamic Inactivation Studies}

The potential efficacy of DIMPy-BODIPY as a photodynamic inactivator of viral agents was tested using three viruses from different families: The enveloped viruses dengue virus type I (Flaviviridae) and vesicular stomatitis virus (Paramyxoviridae), and the non-enveloped virus human adenovirus-5 (Adenoviridae). Viruses were incubated with different concentrations of DIMPy-BODIPY and illuminated $\left(400-700 \mathrm{~nm}, 65 \pm 5 \mathrm{~mW} / \mathrm{cm}^{2}\right)$ as described or kept in the dark as a negative control. Virus infectivity after treatment was determined by plaque (VSV) or immunofocus assay (dengue, HAd-5) on Vero cells (Figure 2). In the absence of illumination, the infectivities of dengue and VSV were not affected at any of the concentrations of photosensitizer tested (Figure 2A). In contrast, after illumination, dengue virus (initially at $6.5 \times 10^{6} \mathrm{FFU} / \mathrm{mL}$ ) was inactivated below the level of detection at DIMPy-BODIPY concentrations of $1 \mu \mathrm{M}$ and $0.1 \mu \mathrm{M}$, which represents a drop in infectivity of over $6 \log$ units reduction in FFU/mL $(p<0.001)$. Even at the low concentration of $0.01 \mu \mathrm{M}$ DIMPy-BODIPY, 
dengue was partially inactivated, with the infectivity showing a reduction of approximately $2 \log$ units in $\mathrm{FFU} / \mathrm{mL}(p<0.001)$.

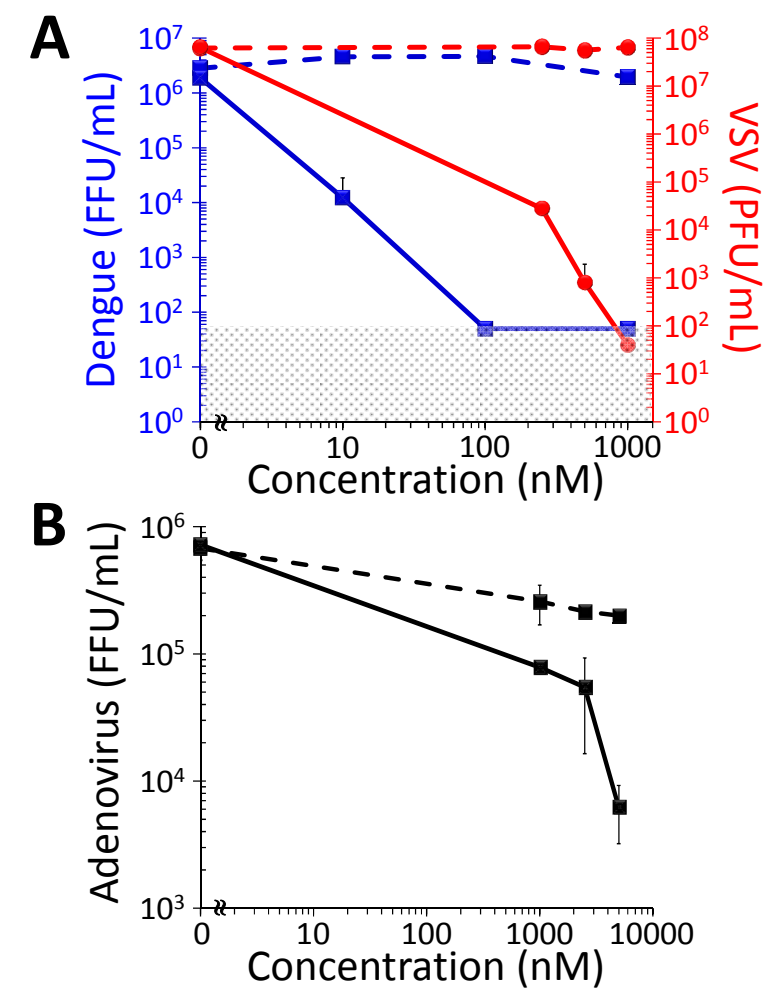

Figure 2. Photodynamic inactivation as a function of DIMPy-BODIPY concentration of (A) dengue 1 virus (blue) and vesicular stomatitis virus (VSV, red); and (B) human adenovirus-5 (HAd-5). In both panels, the solid lines represent the light treated samples, whereas the dashed lines represent the dark controls. For these studies, the illumination conditions were as follows: $30 \mathrm{~min}, 400-700 \mathrm{~nm}, 65 \pm 5 \mathrm{~mW} / \mathrm{cm}^{2}$ (total fluence of $118 \mathrm{~J} / \mathrm{cm}^{2}$ ). The detection limits of the assays were $50 \mathrm{FFU} / \mathrm{mL}$ for the dengue virus study, $40 \mathrm{PFU} / \mathrm{mL}$ for the VSV study, and $66 \mathrm{FFU} / \mathrm{mL}$ for the HAd-5 study; data points below the detection limit were set to these values (represented by the shaded area) for graphing purposes. In the cases where error bars cannot be visualized, the error bars themselves were smaller than the marker employed in the plot.

Pilot experiments demonstrated that VSV proved to be slightly more resistant to inactivation than dengue at $0.1 \mu \mathrm{M}$ DIMPy-BODIPY, remaining almost fully infectious (unpublished data). Therefore, for VSV the concentrations of DIMPy-BODIPY were varied from 0.25 to $1 \mu \mathrm{M}$. As with dengue, the infectivity of VSV was not negatively affected at any of the DIMPy-BODIPY concentrations tested in the absence of illumination. After light treatment, however, VSV (initially at $10^{7} \mathrm{PFU} / \mathrm{mL}$ ) was completely inactivated at $1 \mu \mathrm{M}$ DIMPy-BODIPY, representing a drop in infectivity of over $6 \log$ units reduction in PFU/mL $(p<0.001$; Figure 2A). At $0.5 \mu \mathrm{M}$ DIMPy-BODIPY, the viral infectivity was reduced nearly $5 \log$ units in PFU/mL $(p<0.001)$, and was similarly reduced by nearly $4 \log$ units in $\mathrm{PFU} / \mathrm{mL}(p<0.001)$ at the lowest effective concentration tested of $0.25 \mu \mathrm{M}$ DIMPy-BODIPY.

In contrast to efficient photoinactivation of the enveloped viruses above, the non-enveloped human adenovirus-5 (initially at $6.5 \times 10^{5} \mathrm{FFU} / \mathrm{mL}$ ) proved more resistant to inactivation by DIMPy-BODIPY. 
At $1 \mu \mathrm{M}$, a $1 \log$ unit reduction in FFU/mL $(p<0.025)$ was observed that increased to $2 \log$ units $(p<0.001)$ at a photosensitizer concentration of $5 \mu \mathrm{M}$. A possible explanation to the greater resistance of adenovirus to photoinactivation is that the virus capsid in non-enveloped viruses, which in HAd-5 is comprised of at least nine different proteins (32), affords a greater level of protection to the virion from its surrounding environment than the lipid membrane and associated proteins of enveloped ones. Interestingly, the dark control samples themselves showed $\sim 80 \%$ reduction in $\mathrm{FFU} / \mathrm{mL}$ for $1-5 \mu \mathrm{M}$ DIMPy-BODIPY, suggesting a non-photoinduced virus inactivation pathway may also be present, but this was not further explored. Finally, red blood cell hemolysis assays showed no statistically significant hemolytic property associated with DIMPy-BODIPY when tested to $10 \mu \mathrm{M}$ concentration (Supplementary Table S1).

\subsection{Antibacterial Photodynamic Inactivation Studies}

In vitro aPDI studies employing the photosensitizer DIMPy-BODIPY were performed in a concentration-dependent manner. The illumination time was fixed at $30 \mathrm{~min}$ for the bacterial studies as determined by a time-dependence study of the photobleaching of DIMPy-BODIPY (see Supplementary Data and Figure S1). All studies with bacteria employed a starting concentration of $1-4 \times 10^{8} \mathrm{CFU} / \mathrm{mL}$ as determined by colony counting. For the three Gram-positive bacteria S. aureus ATCC-2913, methicillin-resistant S. aureus (MRSA) strain ATCC-44, and the vancomycin-resistant E. faecium (VRE) strain ATCC-2320, they were found to be highly susceptible to photodynamic inactivation with DIMPy-BODIPY regardless of their respective antibiotic resistance (Figure 3A). For example, at the concentration of $0.1 \mu \mathrm{M}$, DIMPy-BODIPY reduced bacterial survival by 5-6 log units $(99.999+\%$ viable cell eradication, $p<0.001)$ for all three after $30 \mathrm{~min}$ illumination $\left(400-700 \mathrm{~nm}, 65 \pm 5 \mathrm{~mW} / \mathrm{cm}^{2}\right)$. At concentrations above $0.25 \mu \mathrm{M}$ under the same illumination conditions, no surviving bacteria were detected for any of the three bacteria. By comparison, the benchmark photosensitizers TMPyP (Supplementary Figure S3A) and methylene blue (Supplementary Figure S4A) showed no statistically significant cell inactivation at $0.1 \mu \mathrm{M}$ photosensitizer concentration, and were only able to achieve a $5 \log$ units reduction in bacteria when 5- to 10-fold higher concentrations of the photosensitizers were employed (typically $1 \mu \mathrm{M}$ or higher). See Supplementary Figure S5 for a comparison of their electronic absorption spectra and that of DIMPy-BODIPY. Decreasing the DIMPy-BODIPY concentration to $0.05 \mu \mathrm{M}$ led to a partial attenuation of the inactivation efficacy for both $S$. aureus ATCC-2913 (2.5 log units reduction in CFU/mL, $p<0.001$ ) and E. faecium ATCC-2320 (3.5 log units reduction, $p<0.001$ ), whereas there was no statistical difference observed for the MRSA strain ATCC-44 between 0.05 and $0.1 \mu \mathrm{M}$ concentrations (both $\sim 5 \log$ units reduction). When the concentration of the photosensitizer was further decreased to $0.025 \mu \mathrm{M}$, each of the $S$. aureus strains ceased to be inactivated, while the E. faecium strain remarkably still exhibited $99 \%$ inactivation (2 $\log$ units, $p<0.001$ ). No statistically significant inactivation was seen at a DIMPy-BODIPY concentration of $0.01 \mu \mathrm{M}$ for any of the three aforementioned bacteria.

The taxonomically Gram-positive bacterium M. smegmatis strain ATCC-700084, shown separately in Figure 4, exhibited $\sim 4 \log$ units inactivation at $1 \mu \mathrm{M}(p<0.001), \sim 3.3 \log$ units at $0.5 \mu \mathrm{M}(p<0.001)$, and $95 \%$ inactivation $(\sim 2 \log$ units, $p<0.001)$ at $0.25 \mu \mathrm{M}$. No statistically significant inactivation of this M. smegmatis strain was observed at DIMPy-BODIPY concentrations below $0.1 \mu \mathrm{M}$. By comparison, 
methylene blue is a much poorer photosensitizer under identical illumination conditions, having previously been shown to photoinactivate $M$. smegmatis by $4 \log$ units at only a very high concentration of $75 \mu \mathrm{M}$, by $2 \log$ units at $7.5 \mu \mathrm{M}$, and showed no inactivation at $0.75 \mu \mathrm{M}$ [13]. However, under the same conditions, TMPyP has been shown to be highly efficient in its photoinactivation of M. smegmatis, achieving detection limit inactivation (6+ log units reduction) at concentrations as low as $0.15 \mu \mathrm{M}[13]$.
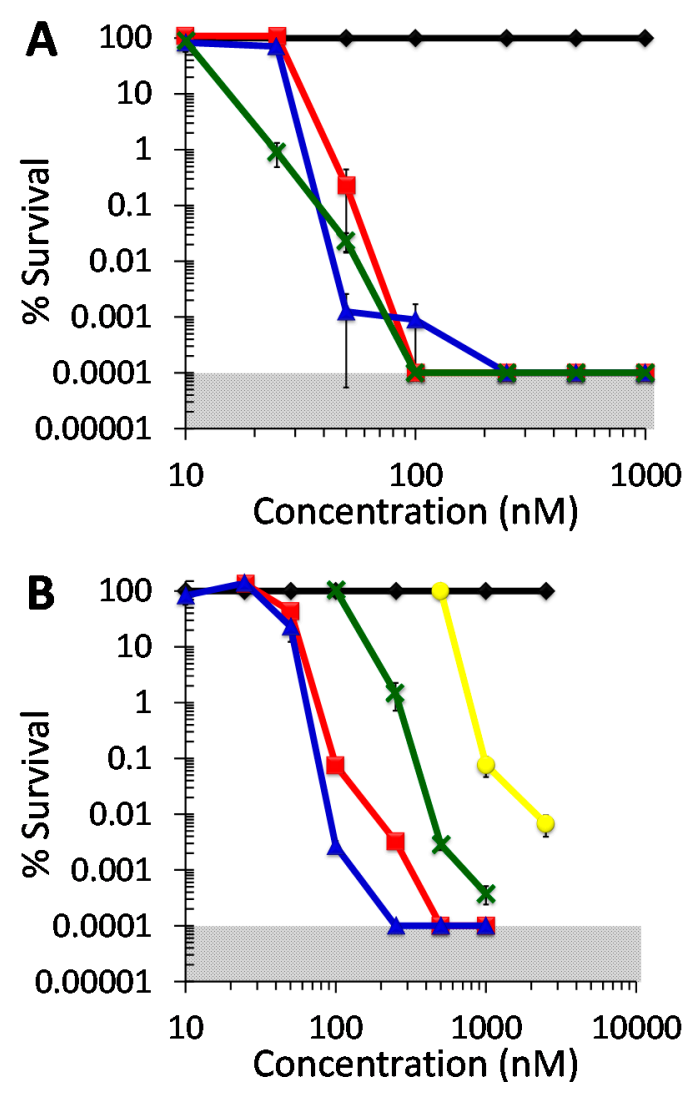

Figure 3. Photodynamic inactivation of bacteria as a function of DIMPy-BODIPY concentration. (A) Gram positive species. Displayed is the \% survival of the dark control $(\diamond)$ and the light treated samples for methicillin-susceptible S. aureus (MSSA) ATCC-2913 (घ), methicillin-resistant S. aureus (MRSA) ATCC-44 ( $\mathbf{\Delta})$, and the vancomycin-resistant Enterococcus faecium (VRE) ATCC-2320 strain (x). (B) Gram negative species. Displayed is the \% survival of the dark control $(\diamond)$ and the light treated samples for A. baumannii

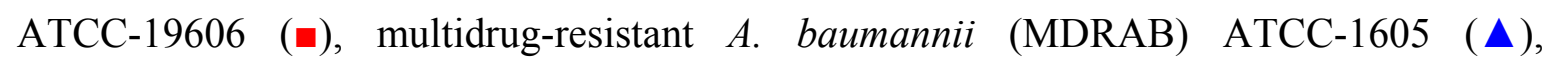
P. aeruginosa ATCC-97 (x), and K. pneumoniae ATCC-2146 (॰). For all bacteria, the illumination conditions were as follows: $30 \mathrm{~min}, 400-700 \mathrm{~nm}, 65 \pm 5 \mathrm{~mW} / \mathrm{cm}^{2}$ (total fluence of $118 \mathrm{~J} / \mathrm{cm}^{2}$ ). As the plating technique employed to determine $\%$ survival did not allow for detection of survival rates of $<0.0001 \%$, data points below the detection limit were set to $0.0001 \%$ survival for graphing purposes. The shaded areas correspond to undetectable cell survival with the assay employed. In the cases where error bars cannot be visualized, the error bars themselves were smaller than the marker employed in the plot. 


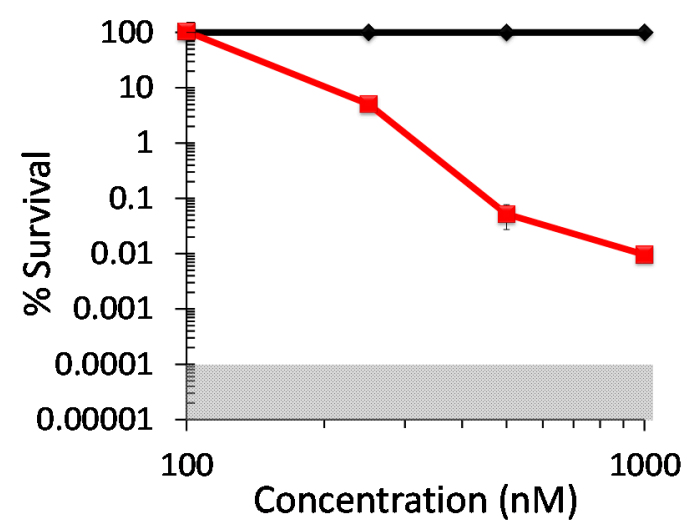

Figure 4. Photodynamic inactivation of Mycobacterium smegmatis $\mathrm{mc}^{2} 155$ ATCC-700084 as a function of DIMPy-BODIPY concentration. Displayed is the $\%$ survival of the dark control $(\diamond)$ and the light treated samples $(\square)$. Illumination and assay conditions were as in Figure 3.

While the Gram-positive species were fairly consistent with respect to their photoinactivation when compared to one another, the Gram-negative ones (drug-susceptible A. baumannii strain ATCC-19606, the multi-drug resistant $A$. baumannii (MDRAB) strain ATCC-1605, P. aeruginosa strain ATCC-97 and $K$. pneumoniae strain ATCC-2146) had a wider range of susceptibilities to BODIPY-mediated aPDI (Figure 3B). When the efficacy of DIMPy-BODIPY at a concentration of $0.5 \mu \mathrm{M}$ was examined against the two $A$. baumannii strains, no CFUs were detected, and survival rates were therefore below the detection limit of $<0.0001 \%$, corresponding to an impressive $6 \log$ units reduction in viable cells $(p<0.001)$. At $0.1 \mu \mathrm{M}$, DIMPy-BODIPY was still able to achieve a noteworthy 3 and $4.5 \log$ units reduction in CFU for A. baumannii ATCC-19606 and MDRAB ATCC-1605, respectively, whereas $0.05 \mu \mathrm{M}$ was the lowest PS concentration for which aPDI was statistically significant (57\% and 77\% cell eradication for A. baumannii ATCC-19606 and MDRAB ATCC-1605, respectively, $p<0.02$ ). No statistically significant inactivation of either strain was seen at a DIMPy-BODIPY concentration of $0.025 \mu \mathrm{M}$ or lower. Thus, with the exceptions at the extremes of the concentrations studied (above $0.5 \mu \mathrm{M}$ or below $0.025 \mu \mathrm{M}$ ), the MDRAB ATCC-1605 strain was statistically more susceptible ( $p=\sim 0.02$ or lower), albeit only slightly, to photodynamic inactivation by DIMPy-BODIPY when compared with A. baumannii ATCC-19606. By comparison, the commercial photosensitizers were poorer performers: TMPyP (Supplementary Figure S3B) required a 5-fold higher concentration $(2.5 \mu \mathrm{M})$ to achieve $5 \log$ units of inactivation, and methylene blue (Supplementary Figure S4B) required a 2 -fold higher concentration $(1.0 \mu \mathrm{M})$, with both exhibiting no statistically significant photoinactivation at or below $0.25 \mu \mathrm{M}$.

The $P$. aeruginosa ATCC-97 strain was also found to be inactivated by DIMPy-BODIPY at sub- $\mu \mathrm{M}$ to low $\mu \mathrm{M}$ concentrations (Figure 3B): $\sim 5.5 \log$ units inactivation was observed $(p<0.001)$ at a photosensitizer concentration of $1 \mu \mathrm{M}$. Lowering the concentration to the sub $\mu \mathrm{M}$ regime decreased the inactivation efficacy; however, it was still possible to achieve a notable $\sim 4.5 \log$ units reduction in $\mathrm{CFU} / \mathrm{mL}$ at $0.5 \mu \mathrm{M}(p<0.001)$, and $98 \%$ inactivation $(\sim 2 \log$ units, $p<0.001)$ at $0.25 \mu \mathrm{M}$. No statistically significant inactivation of this $P$. aeruginosa strain was observed at DIMPy-BODIPY concentrations below $0.1 \mu \mathrm{M}$. As a benchmark, methylene blue was able to match the highest observed level of photoinactivation $(\sim 5.5 \log$ units; Supplementary Figure S4B), but required a 5-fold higher 
photosensitizer concentration $(5 \mu \mathrm{M})$ to do so, whereas TMPyP (Supplementary Figure S3B) was only able to achieve $3 \log$ units reduction in viable cells at that same concentration.

In comparison to the other bacterial species investigated, the multi-drug resistant NDM-1-producing K. pneumoniae clinical isolate ATCC-2146 explored here was the least susceptible to photodynamic inactivation by DIMPy-BODIPY, with no inactivation observed at $0.5 \mu \mathrm{M}$ (Figure 3B). At $1 \mu \mathrm{M}$, a concentration that achieved a detection-limit level of inactivation for nearly all other bacteria examined, a 99.9\% (3 log units) reduction in CFU/mL was obtained for this $K$. pneumoniae strain $(p<0.001)$. In an attempt to see if near detection limit inactivation was possible, the photosensitizer concentration was increased to $2.5 \mu \mathrm{M}$, which showed $\sim 4 \log$ units of inactivation ( $>99.99 \%$, $p<0.001)$. By comparison, TMPyP was slightly more effective than DIMPy-BODIPY, reaching $\sim 5 \log$ units reduction at $2.5 \mu \mathrm{M}$ (Supplementary Figure S3B), whereas methylene blue was a far poorer photosensitizer, exhibiting no statistically significant photoinactivation at that concentration (Supplementary Figure S4B).

The two main pathways by which aPDI inactivates microbes include: The Type I mechanism (redox), where the photosensitizer in its triplet state undergoes electron transfer reactions with suitable substrates such as cellular biomolecules, solvent molecules or dioxygen, forming cytotoxic free radicals and superoxide; and the Type II mechanism (energy transfer), where energy transfer from the excited triplet state of the photosensitizer to ground state triplet molecular oxygen leads to the formation of highly reactive singlet oxygen [36]. In order to probe whether DIMPy-BODIPY mediates aPDI through, in part, a Type II mechanism for photosensitization, the singlet oxygen quantum yield was measured in methanol by direct observation of the singlet oxygen phosphorescence, and was found to be $11 \%$. This is in comparison with the $\Phi=19 \%$ value previously reported in isopropanol [26]. Moreover, the known singlet oxygen quencher sodium azide was employed in cell survival studies [13]. When the aPDI assay was repeated for $A$. baumannii in the presence of $0.2-20 \mathrm{mM} \mathrm{NaN}_{3}$, a statistically significant increase in cell survival was observed for DIMPy-BODIPY (Supplementary Figure S2, Supplementary Data), suggesting that aPDI of A. baumannii (ATCC-19606) is mediated in part by singlet oxygen production. Cell survival was increased at the sodium azide concentration of $0.2 \mathrm{mM}$, and the greatest increase was noted at $20 \mathrm{mM}$ : Specifically, only $3 \log$ units of inactivation were observed in the presence of the singlet oxygen quencher vs. $6 \log$ units in its absence. Above $20 \mathrm{mM}$, control experiments showed the azide inhibited growth of the bacterium (unpublished data), and for this reason higher concentrations of azide were not pursued due to its toxicity as a metabolic inhibitor. Although the data strongly suggest a Type II mechanism of aPDI by DIMPy-BODIPY, contributions from a Type I pathway cannot be ruled out.

Despite knowing that in situ formed singlet oxygen is the likely microbiocidal reagent, we are unable to determine if strain-specific variances or species-specific differences are responsible for the different aPDI inactivation efficiencies observed for the Gram-negative bacteria investigated in this study. Given that the photoinactivation of the Gram-positive bacteria was fairly consistent, one obvious factor may be different compositions and properties associated with the bacterial outer membrane of the Gram-negative bacteria. This reasoning could explain, for example, why M. smegmatis was more difficult to photoinactivate when compared to $A$. baumannii or $P$. aeruginosa given that its cell wall is thicker than in many other bacteria, as well as more hydrophobic and waxy due to it being rich in mycolic acids/mycolates [37]. Different liposaccharide compositions could also result in variations of the 
number/density of negative charges present in the outer membrane, which in turn would affect cationic photosensitizer binding, whereas differences in the porins may also affect photosensitizer uptake. Finally, variations in the number and efficiency of efflux pumps, particularly important for antibiotic resistance in $K$. pneumoniae [38], may also be a factor in expelling photosensitizers that may be uptaken by the bacteria.

\subsection{Antifungal Photodynamic Inactivation Studies}

Two opportunistic Candida pathogens, the C. albicans strain ATCC-90028 (Figure 5A) and the C. glabrata strain ATCC-15545 (Figure 5B), proved to be similar in their susceptibilities to photoinactivation with DIMPy-BODIPY. Each strain, initially at $\sim 10^{7} \mathrm{CFU} / \mathrm{mL}$, showed detection limit inactivation (5 log units CFU reduction) with $5 \mu \mathrm{M}$ DIMPy-BODIPY and either 15 or 30 min of illumination $(p<0.001)$.
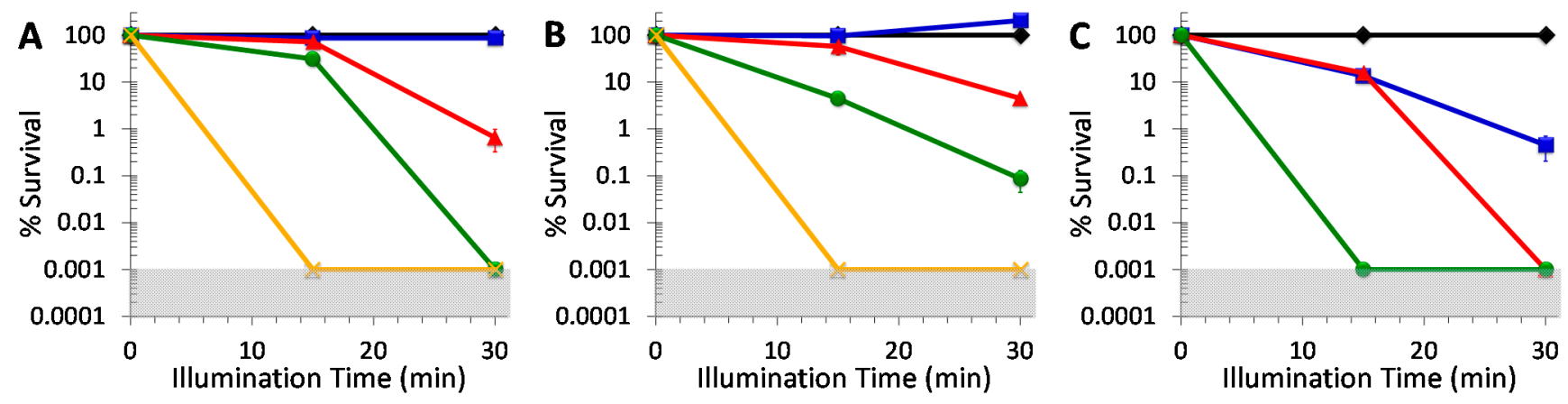

Figure 5. Photodynamic inactivation of (A) Candida albicans ATCC-90028, (B) Candida glabrata ATCC-15545, and (C) Cryptococcus neoformans ATCC-64538 as a function of DIMPy-BODIPY concentration and illumination time. Displayed is the \% survival of the dark control $(\diamond)$ and the light treated samples at $0.1 \mu \mathrm{M}(\bullet), 0.5 \mu \mathrm{M}(\Delta), 1 \mu \mathrm{M}(\bullet)$, and $5 \mu \mathrm{M}(\mathrm{X})$. Illumination conditions were as follows: 400-700 nm, $65 \pm 5 \mathrm{~mW} / \mathrm{cm}^{2}$, and either 15 or $30 \mathrm{~min}$ (total fluences of 59 and $118 \mathrm{~J} / \mathrm{cm}^{2}$, respectively). As the plating technique employed to determine $\%$ survival did not allow for detection of survival rates of $<0.001 \%$, data points below the detection limit were set to $0.001 \%$ survival for graphing purposes. The shaded areas correspond to undetectable cell survival with the assay employed. In the cases where error bars cannot be visualized, the error bars themselves were smaller than the marker employed in the plot.

For studies performed at $1 \mu \mathrm{M}$ concentration, the $C$. albicans strain exhibited $69 \%$ cell inactivation after 15 min of illumination $(p<0.001)$, and proved highly susceptible upon an increase in illumination time, with a full $5 \log$ units of inactivation to the detection limit with 30 min illumination. The C. glabrata strain was $95 \%(p<0.001)$ inactivated after 15 min illumination, though it only exhibited a slight increase to $3 \log$ units inactivation $(p<0.001)$ at the longer 30 min illumination time. The C. albicans strain $(99.5 \%, \sim 2 \log$ units, $p<0.001)$ was also inactivated more efficiently than the C. glabrata one $(95 \%, \sim 1 \log$ unit, $p<0.001)$ at $30 \mathrm{~min}$ of illumination and $0.5 \mu \mathrm{M}$ DIMPy-BODIPY concentration. No inactivation of the two Candida strains was observed for either 15 or 30 min illumination at a photosensitizer concentration of $0.1 \mu \mathrm{M}$. Overall, these results with 
DIMPy-BODIPY compared favorably against the commercial photosensitizer methylene blue (for C. albicans, no inactivation was observed with $1 \mu \mathrm{M}$ methylene blue, and $\sim 4.5 \log$ units at $5 \mu \mathrm{M}$ PS concentration (Supplementary Figure S6A); for C. glabrata, $\sim 3 \log$ units reduction at $5 \mu \mathrm{M}$ (Supplementary Figure S7A), and were comparable to the detection limit inactivation observed for TMPyP at $5 \mu \mathrm{M}$ using either 15 or 30 min of illumination for C. albicans (Supplementary Figure S6B) and C. glabrata (Supplementary Figure S7B).

The yeast pathogen Cryptococcus neoformans ATCC-64538, whose species gives rise to an estimated one million cases of meningitis resulting in 625,000 deaths worldwide each year [39], proved to be more susceptible to photoinactivation with DIMPy-BODIPY than either of the Candida species (Figure 5C). Starting with an initial concentration of $\sim 10^{7} \mathrm{CFU} / \mathrm{mL}$, detection limit inactivation (5 log units CFU reduction) was achieved at a photosensitizer concentration of $1 \mu \mathrm{M}$ with 15 min illumination, and at $0.5 \mu \mathrm{M}$ with the longer 30 min illumination $(p<0.001)$. Remarkably, even at the lowest concentration examined of $0.1 \mu \mathrm{M}$ DIMPy-BODIPY, a noteworthy $99.5 \%(2+\log$ units, $p<0.001)$ reduction in CFU was observed for this $C$. neoformans strain for the 30 min illumination time. Again, these results for DIMPy-BODIPY compare favorably against the benchmark photosensitizers methylene blue (Supplementary Figure S8A) and TMPyP (Supplementary Figure S8B), both of which were able to reach detection limit inactivation, but required a 5-fold higher concentration of $5 \mu \mathrm{M}$ to do so when compared with DIMPy-BODIPY.

The difference in photoinactivation efficacy between the three different yeast strains may be attributed to differences in their extracellular structures. C. neoformans is encapsulated and known to produce melanin, while Candida species do not. The capsule contains highly negative charged polysaccharides found immediately outside the cell wall [40-42], namely glucuronoxylomannan (GXM), galactoxylomannan (GalXM) and mannoprotein, where GXM makes up approximately 90\% of the capsule composition and ranges from 1 to $50 \mu \mathrm{m}$ in thickness [43]. Both the polysaccharide and melanin production result in a strong negative charge on the cell surface. The high photodynamic inactivation efficacy of the cationic DIMPy-BODIPY towards C. neoformans may be explained by a stronger electrostatic interaction between the cell surface and the photosensitizer than with the Candida species.

\section{Experimental Section}

\subsection{Materials}

Buffer salts and methylene blue were purchased from Fisher Scientific (Pittsburgh, PA, USA) Nutrient Broth \#234000 was obtained from BD Difco (Franklin Lakes, NJ, USA), LB broth Miller from EMD Chemicals (Billerica, MA, USA), tetramethylpyridylporphyrin tetratosylate from Frontier Scientific, and Tryptic Soy Broth from Teknova (Hollister, CA, USA). Polylysine solution was purchased from Sigma Aldrich (St. Louis, MO, USA). Unless otherwise specified, all other chemicals were obtained from commercial sources in the highest purity available. Ultrapure water used for all media and buffers was provided by an Easypure II system (Barnstead, Dubuque, IA, USA). UV-visible absorption measurements were performed on a Cary 50 Bio instrument (Varian, Santa Clara, CA, USA) or a Genesys 10 UV scanning spectrophotometer from Thermo Electron Corp. (Waltham, MA, 
USA) for single wavelength measurements. The photosensitizer 2,6-diiodo-1,3,5,7-tetramethyl-8-( $N$ methyl-4-pyridyl)-4,4'-difluoroboradiazaindacene [DIMPy-BODIPY; $\log p=-1.96 ; \lambda_{\max }\left(\mathrm{H}_{2} \mathrm{O}\right)=509 \mathrm{~nm}$ $\left.\left(75.9 \mathrm{mM}^{-1} \cdot \mathrm{cm}^{-1}\right)\right]$ was synthesized per the published protocol $[26,30,44]$.

\subsection{Cell Culture}

All bacteria were grown in $5 \mathrm{~mL}$ cultures incubated at $37{ }^{\circ} \mathrm{C}$ on an orbital shaker under the following growth conditions: Acinetobacter baumannii (ATCC-19606) was grown in Miller-LB media without antibiotics; the multi-drug resistant strain of Acinetobacter baumannii (ATCC-1605) was grown in Miller-LB media with $5 \mu \mathrm{g} / \mathrm{mL}$ tetracycline; methicillin-susceptible Staphylococcus aureus 2913 was grown in tryptic soy broth media without antibiotics; methicillin-resistant Staphylococcus aureus (ATCC-44) was grown in tryptic soy broth media with $5 \mu \mathrm{g} / \mathrm{mL}$ tetracycline; Pseudomonas aeruginosa (ATCC-97) was grown in BD Difco Nutrient Broth \#234000 with $5 \mu \mathrm{g} / \mathrm{mL}$ tetracycline; Mycobacterium smegmatis $\mathrm{mc}^{2} 155$ was grown in BD Difco $7 \mathrm{H} 9$ media with ADS and $100 \mu \mathrm{g} / \mathrm{mL}$ cycloheximide; Klebsiella pneumoniae (ATCC-2146) was grown in BD Difco Nutrient Broth \#234000 with $100 \mu \mathrm{g} / \mathrm{mL}$ ampicillin. The vancomycin-resistant strain of Enterococcus faecium (ATCC-2320) was grown in BD Difco Bacto Brain Heart Infusion 237,500 with $100 \mu \mathrm{g} / \mathrm{mL}$ ampicillin. Each bacterium was grown to a concentration of $1-4 \times 10^{8} \mathrm{CFU} / \mathrm{mL}$ (determined spectrophotometrically from growth curves using a Genesys $10 \mathrm{UV}$ scanning spectrophotometer) prior to being pelleted by centrifugation (10 min, $\sim 3700 \mathrm{~g}$ ). Once pelleted, the supernatant was decanted and the cells were resuspended in $5 \mathrm{~mL}$ of PBS $\left(170 \mathrm{mM} \mathrm{NaCl}, 3.4 \mathrm{mM} \mathrm{KCl}, 10.0 \mathrm{mM} \mathrm{Na} 2 \mathrm{HPO}_{4}, 1.8 \mathrm{mM} \mathrm{KH}_{2} \mathrm{PO}_{4}\right.$, $\mathrm{pH}$ 7.2) containing $0.05 \%$ Tween-80 (to prevent agglomeration of $M$. smegmatis [45]) and diluted to $\sim 10^{8} \mathrm{CFU} \cdot \mathrm{mL}^{-1}$ (determined spectrophotometrically). Candida albicans (ATCC-90028) and Candida glabrata (ATCC-15545) were grown aerobically overnight in yeast extract-peptone-dextrose (YPD) broth at $37{ }^{\circ} \mathrm{C}$. Cryptococcus neoformans (ATCC-64538) was grown aerobically in Sabouraud dextrose broth at $30{ }^{\circ} \mathrm{C}$ for $48 \mathrm{~h}$. Cells were harvested by centrifugation $(10 \mathrm{~min}, \sim 3700 \mathrm{~g})$ and washed twice with PBS. The cells were resuspended in PBS and diluted to $\sim 10^{7} \mathrm{CFU} \cdot \mathrm{mL}^{-1}$ (determined spectrophotometrically).

\subsection{Viral Propagation}

Vesicular stomatitis virus (VSV) NJ strain was propagated on Vero cells and titered by plaque assay on Vero cells. Dengue 1 virus was propagated on C6/36 mosquito cells and titered on Vero cells by immunofocus assay. Human adenovirus-5 (HAd-5) was propagated on the human lung carcinoma cell line A549 and titered on the same cells.

\subsection{Photodynamic Inactivation Assay}

All photosensitization experiments were performed using a non-coherent light source, PDT light model LC122 (LumaCare, Newport Beach, CA, USA), equipped with a LUM V fiber optic probe (400-700 $\mathrm{nm}$ band pass filter, average transmittance $\mathrm{T}_{\mathrm{avg}} \sim 95 \% \pm 3 \%$ ) and an OSRAM Xenophot lamp model 64653 HLX (24 V, $250 \mathrm{~W})$. The fluence rate was measured with an Orion power meter (Orphir Optronics Ltd, Jerusalem, Israel). All experiments were conducted in triplicate at a minimum, and 
statistical significance was assessed via a two-tailed, unpaired Student's $t$-test. Sterile stock solutions of DIMPy-BODIPY were prepared in filter sterilized ultrapure water.

\subsubsection{Bacteria and Yeast}

Five $\mathrm{mL}$ cultures were incubated with DIMPy-BODIPY $(0.01-2.5 \mu \mathrm{M}$ final concentration as indicated in Figures 3 and 4 for bacteria, and $0.01-5 \mu \mathrm{M}$ final concentration for yeast) on an orbital shaker in the dark for 5 (bacteria) or 15 (yeast) min. After incubation, three $1 \mathrm{~mL}$ aliquots of the cell suspension were transferred to a sterile 24-well plate (BD Falcon, flat bottom) and illuminated with visible light $(400-700 \mathrm{~nm})$ with a fluence rate of $65 \pm 5 \mathrm{~mW} / \mathrm{cm}^{2}$ for a variable period of time (5-60 min) while magnetically stirred. The remaining aliquots of cell culture were kept in the absence of light as the dark control. Studies were repeated in the absence of the photosensitizer as a no compound light control (Supplementary Table S2). After illumination, each well was 1:10 serially diluted five times. $10 \mu \mathrm{L}$ from the undiluted well and from each dilution, as well as from the dark control, were plated and incubated in the dark at $37^{\circ} \mathrm{C}$. Each bacterium was grown on gridded six column square agar plates made with their respective growth media without antibiotics, with the exception of M. smegmatis, which was plated on BD Difco 7H10-ADS containing $100 \mu \mathrm{g} / \mathrm{mL}$ cycloheximide. The survival rate was determined from the ratio of CFU $/ \mathrm{mL}$ of the illuminated solution $v s$. that of the dark control. The minimum detection limit was $100 \mathrm{CFU} / \mathrm{mL}$ (based on $10 \mu \mathrm{L}$ plated from the $1 \mathrm{~mL}$ undiluted well). Variations in the concentration of the starter culture $\left(1-4 \times 10^{8} \mathrm{CFU} / \mathrm{mL}\right.$ for bacteria, and $1-4 \times 10^{7} \mathrm{CFU} / \mathrm{mL}$ for yeast) resulted in a variation of the detection limit spanning the region of $0.001 \%-0.0001 \%$ survival for bacteria, and $0.01 \%-0.001 \%$ for yeast, respectively. Samples with PS present but kept in the dark (dark control) and illuminated samples without PS (light control) served as controls.

\subsubsection{Vesicular Stomatitis Virus}

VSV ( $10^{7}$ plaque forming units, PFU) of were incubated with DIMPy-BODIPY $(0.01-1 \mu \mathrm{M}$ final concentration) for $5 \mathrm{~min}$ in the dark prior to $30 \mathrm{~min}$ under visible light illumination $(400-700 \mathrm{~nm}$; $65 \pm 5 \mathrm{~mW} / \mathrm{cm}^{2}$ ) in a total volume of $100 \mathrm{~mL}$ of MEM supplemented with $10 \mathrm{mM}$ HEPES, $1 \%$ FBS and antibiotics. Control experiments were similarly performed in the dark. Aliquots of virus samples were subsequently titered on Vero cells, and the virus concentration was determined by plaque assay (detection limit of $40 \mathrm{PFU} / \mathrm{mL}$ ). Specifically, samples were set up in biological duplicates, viruses were titered by serial 10 -fold dilution on Vero cells in 24 -well plates at $37{ }^{\circ} \mathrm{C}$. Plaques were detected by crystal violet staining $48 \mathrm{~h}$ after infection. Where virus was detectable, the plaques at dilutions where wells contained between 10 to 20 plaques were counted for titer determination. At concentrations of DIMPy-BODIPY where the virus sample was largely inactivated it was necessary to use wells containing fewer plaques. The limit of detection was $40 \mathrm{PFU} / \mathrm{mL}$.

\subsubsection{Dengue-1 Virus}

The virus $\left(6.5 \times 10^{6}\right.$ focus forming units, FFU) was incubated with DIMPy-BODIPY $(0.25-1 \mu \mathrm{M}$ final concentration) for $5 \mathrm{~min}$ in the dark prior to $30 \mathrm{~min}$ under visible light illumination (400-700 $\mathrm{nm}$; 
$65 \pm 5 \mathrm{~mW} / \mathrm{cm}^{2}$ ) in a total volume of $100 \mathrm{~mL}$ of MEM supplemented with $10 \mathrm{mM}$ HEPES, $1 \%$ FBS and antibiotics. Control experiments were similarly performed in the dark. Samples set up in biological triplicate were used. Virus was titered by serial 10-fold dilution on A549 cells in 24-well plates at $37{ }^{\circ} \mathrm{C}$ for $72 \mathrm{~h}$. Immunofoci were detected with an antibody to the E1A protein and a secondary anti-mouse antibody conjugated to horseradish peroxidase. Where virus was detectable, immunofoci at dilutions where wells contained between 10 to 20 foci were counted for titer determination. At concentrations of DIMPy-BODIPY where the virus sample was largely inactivated it was necessary to use wells containing fewer foci. The detection limit was $50 \mathrm{FFU} / \mathrm{mL}$.

\subsubsection{Human Adenovirus-5}

The virus $\left(6.5 \times 10^{5}\right.$ focus forming units, FFU) was incubated with DIMPy-BODIPY $(0.25-1 \mu \mathrm{M}$ final concentration) for $5 \mathrm{~min}$ in the dark prior to $30 \mathrm{~min}$ under visible light illumination $(400-700 \mathrm{~nm}$; $65 \pm 5 \mathrm{~mW} / \mathrm{cm}^{2}$ ) in a total volume of $100 \mathrm{~mL}$ of MEM supplemented with $10 \mathrm{mM}$ HEPES, $1 \%$ FBS and antibiotics. Control experiments were similarly performed in the dark. Samples set up in biological triplicate were used. Virus was titered by serial 10-fold dilution on A549 cells in 24-well plates at $37{ }^{\circ} \mathrm{C}$ for $72 \mathrm{~h}$. Immunofoci were detected with an antibody to the E1A protein and a secondary anti-mouse antibody conjugated to horseradish peroxidase. Where virus was detectable, immunofoci at dilutions where wells contained between 10 to 20 foci were counted for titer determination. At concentrations of DIMPy-BODIPY where the virus sample was largely inactivated it was necessary to use wells containing fewer plaques. The detection limit was $66 \mathrm{FFU} / \mathrm{mL}$.

\subsection{Red Blood Cell Hemolysis Assay}

Hemolysis assays employing mechanically defibrinated sheep blood were performed following literature protocol [46], using the following concentrations of DIMPy-BODIPY: 0.05, 0.1, 0.5, 1, 5 and $10 \mu \mathrm{M}$. A compound-free control was performed to verify the background, and a $2 \%(v / v)$ Triton $\mathrm{X}$ in $0.5 \mathrm{~mL}$ PBS was used as the $100 \%$ lysis positive control.

\subsection{Singlet Oxygen Quantum Yield Determination}

Singlet oxygen formation quantum yield measurements were performed in aerated solutions of methanol in a $1 \mathrm{~cm}^{2}$ quartz cell at ambient temperature with an Edinburgh fluorimeter (FS920) and near-IR photomultiplier tube. The resulting value for the singlet oxygen phosphorescence of DIMPy-BODIPY was compared to Rose Bengal $(\Phi=0.80$ [47]) in air-saturated methanol with a $514.5 \mathrm{~nm}$ excitation wavelength at a power of $250 \mathrm{~mW}$. The quantum yield was calculated as previously described [48].

\section{Conclusions}

We have now further investigated the ability of BODIPY-based photosensitizers to photoinactivate bacteria, fungi, and viruses using visible light $(400-700 \mathrm{~nm})$. Although we cannot rule out strain-specific results given the limited numbers of pathogens explored herein, the results obtained demonstrate that DIMPy-BODIPY is able to mediate the photodynamic inactivation of 
clinically-relevant microbes, including Gram-positive, Gram-negative, and drug-resistant bacteria, as well as pathogenic yeast and model viruses, at nanomolar concentrations and short illumination times. With minor exceptions, DIMPy-BODIPY performed more efficiently over the entire range of microbes studied when compared to the benchmark standards of methylene blue or TMPyP, thus highlighting the utility of the BODIPY-class of photosensitizers for a broad spectrum of potential aPDI application, particularly when one considers the possible synthetic modifications to this modular scaffold. In addition to their potential for in vivo antimicrobial photodynamic therapy, BODIPY-based chromophores may represent an additional class of photosensitizers for incorporation in photomicrobiocidal materials for the elimination of pathogens from surfaces prior to their transmission to hosts [49], and future studies are planned to demonstrate the potential of this new class of photosensitizer for application as a more broadly applicable anti-infective agent.

\section{Supplementary Materials}

Illumination time dependence study (Supplementary Figure S1), sodium azide singlet oxygen quenching study (Supplementary Figure S2), red blood cell hemolysis assay (Supplementary Table S1), light control data (Supplementary Table S2), PDI of bacteria by TMPyP (Supplementary Figure S3) and methylene blue (Supplementary Figure S4), electronic absorption spectra of DIMPy-BODIPY, methylene blue, and TMPyP (Supplementary Figure S5), and PDI of yeast by TMPyP and methylene blue (Supplementary Figures S6-S8) are also provided.

Supplementary materials can be accessed at: http://www.mdpi.com/1420-3049/20/06/10604/s1.

\section{Acknowledgments}

M. smegmatis $\mathrm{mc}^{2} 155$ cells were kindly gifted by William Jacobs (Albert Einstein College of Medicine, New York, NY, USA). This research was supported by a North Carolina State University Research Innovation and Seed Funding (RISF) grant \#2014-0793.

\section{Author Contributions}

BC, FS, WW and RG designed the research; BC, XS, FS and JB performed the research and analyzed the data; $\mathrm{BC}$ and $\mathrm{RG}$ wrote the paper. All authors read, edited, and approved the final manuscript.

\section{Conflicts of Interest}

The authors declare no conflict of interest.

\section{References}

1. Vicente, M.; Hodgson, J.; Massidda, O.; Tonjum, T.; Henriques-Normark, B.; Ron, E.Z. The fallacies of hope: Will we discover new antibiotics to combat pathogenic bacteria in time? FEMS Microbiol. Rev. 2006, 30, 841-852.

2. Kraus, C.N. Low hanging fruit in infectious disease drug development. Curr. Opin. Microbiol. 2008, 11, 434-438. 
3. Vera, D.M.; Haynes, M.H.; Ball, A.R.; Dai, T.; Astrakas, C.; Kelso, M.J.; Hamblin, M.R.; Tegos, G.P. Strategies to potentiate antimicrobial photoinactivation by overcoming resistant phenotypes. Photochem. Photobiol. 2012, 88, 499-511.

4. Dai, T.; Fuchs, B.B.; Coleman, J.J.; Prates, R.A.; Astrakas, C.; St Denis, T.G.; Ribeiro, M.S.; Mylonakis, E.; Hamblin, M.R.; Tegos, G.P. Concepts and principles of photodynamic therapy as an alternative antifungal discovery platform. Front. Microbiol. 2012, 3, 1-16.

5. St Denis, T.G.; Dai, T.; Izikson, L.; Astrakas, C.; Anderson, R.R.; Hamblin, M.R.; Tegos, G.P. All you need is light: Antimicrobial photoinactivation as an evolving and emerging discovery strategy against infectious disease. Virulence 2011, 2, 509-520.

6. Dai, T.; Huang, Y.Y.; Hamblin, M.R. Photodynamic therapy for localized infections-State of the art. Photodiagn. Photodyn. Ther. 2009, 6, 170-188.

7. Wainwright, M. 'Safe' photoantimicrobials for skin and soft-tissue infections. Int. J. Antimicrob. Agents 2010, 36, 14-18.

8. Merkel, P.B.; Kearns, D.R. Radiationless decay of singlet molecular oxygen in solution. Experimental and theoretical study of electronic-to-vibrational energy transfer. J. Am. Chem. Soc. 1972, 94, 7244-7253.

9. Jori, G.; Fabris, C.; Soncin, M.; Ferro, S.; Coppellotti, O.; Dei, D.; Fantetti, L.; Chiti, G.; Roncucci, G. Photodynamic therapy in the treatment of microbial infections: Basic principles and perspective applications. Lasers Surg. Med. 2006, 38, 468-481.

10. Tavares, A.; Carvalho, C.M.; Faustino, M.A.; Neves, M.G.; Tome, J.P.; Tome, A.C.; Cavaleiro, J.A.; Cunha, A.; Gomes, N.C.; Alves, E.; et al. Antimicrobial photodynamic therapy: Study of bacterial recovery viability and potential development of resistance after treatment. Mar. Drugs 2010, 8, 91-105.

11. Maisch, T. A new strategy to destroy antibiotic resistant microorganisms: Antimicrobial photodynamic treatment. Mini Rev. Med. Chem. 2009, 9, 974-983.

12. Carpenter, B.L.; Feese, E.; Sadeghifar, H.; Argyropoulos, D.S.; Ghiladi, R.A. Porphyrin-cellulose nanocrystals: A photobactericidal material that exhibits broad spectrum antimicrobial activity. Photochem. Photobiol. 2012, 88, 527-536.

13. Feese, E.; Ghiladi, R.A. Highly efficient in vitro photodynamic inactivation of mycobacterium smegmatis. J. Antimicrob. Chemother. 2009, 64, 782-785.

14. Bertoloni, G.; Rossi, F.; Valduga, G.; Jori, G.; van Lier, J. Photosensitizing activity of water- and lipid-soluble phthalocyanines on escherichia coli. FEMS Microbiol. Lett. 1990, 59, 149-155.

15. Wainwright, M.; Phoenix, D.A.; Marland, J.; Wareing, D.R.A.; Bolton, F.J. A study of photobactericidal activity in the phenothiazinium series. FEMS Immunol. Med. Microbiol. 1997, $19,75-80$.

16. Huang, Y.Y.; Balasubramanian, T.; Yang, E.; Luo, D.; Diers, J.R.; Bocian, D.F.; Lindsey, J.S.; Holten, D.; Hamblin, M.R. Stable synthetic bacteriochlorins for photodynamic therapy: Role of dicyano peripheral groups, central metal substitution ( $2 \mathrm{~h}, \mathrm{Zn}, \mathrm{Pd})$, and cremophor el delivery. Chem. Med. Chem. 2012, 7, 2155-2167.

17. Wainwright, M.; Antczak, J.; Baca, M.; Loughran, C.; Meegan, K. Phenothiazinium photoantimicrobials with basic side chains. J. Photochem. Photobiol. B 2015, in press, doi:10.1016/j.jphotobiol.2014.12.017. 
18. Wainwright, M. In defence of 'dye therapy'. Int. J. Antimicrob. Agents 2014, 44, 26-29.

19. De Menezes, H.D.; Rodrigues, G.B.; Teixeira Sde, P.; Massola, N.S., Jr.; Bachmann, L.; Wainwright, M.; Braga, G.U. In vitro photodynamic inactivation of plant-pathogenic fungi colletotrichum acutatum and colletotrichum gloeosporioides with novel phenothiazinium photosensitizers. Appl. Environ. Microbiol. 2014, 80, 1623-1632.

20. Bacellar, I.O.; Pavani, C.; Sales, E.M.; Itri, R.; Wainwright, M.; Baptista, M.S. Membrane damage efficiency of phenothiazinium photosensitizers. Photochem. Photobiol. 2014, 90, 801-813.

21. Ziessel, R.; Ulrich, G.; Harriman, A. The chemistry of bodipy: A new el dorado for fluorescence tools. New J. Chem. 2007, 31, 496-501.

22. Loudet, A.; Burgess, K. Bodipy dyes and their derivatives: Syntheses and spectroscopic properties. Chem. Rev. 2007, 107, 4891-4932.

23. Hendricks, J.A.; Keliher, E.J.; Wan, D.; Hilderbrand, S.A.; Weissleder, R.; Mazitschek, R. Synthesis of [18f]bodipy: Bifunctional reporter for hybrid optical/positron emission tomography imaging. Angew. Chem. 2012, 51, 4603-4606.

24. Ortiz, M.J.; Agarrabeitia, A.R.; Duran-Sampedro, G.; Bañuelos Prieto, J.; Lopez, T.A.; Massad, W.A.; Montejano, H.A.; García, N.A.; Lopez Arbeloa, I. Synthesis and functionalization of new polyhalogenated bodipy dyes. Study of their photophysical properties and singlet oxygen generation. Tetrahedron 2012, 68, 1153-1162.

25. Banfi, S.; Nasini, G.; Zaza, S.; Caruso, E. Synthesis and photo-physical properties of a series of bodipy dyes. Tetrahedron 2013, 69, 4845-4856.

26. Caruso, E.; Banfi, S.; Barbieri, P.; Leva, B.; Orlandi, V.T. Synthesis and antibacterial activity of novel cationic bodipy photosensitizers. J. Photochem. Photobiol. B 2012, 114, 44-51.

27. Frimannsson, D.O.; Grossi, M.; Murtagh, J.; Paradisi, F.; O’Shea, D.F. Light induced antimicrobial properties of a brominated boron difluoride $(\mathrm{bf}(2))$ chelated tetraarylazadipyrromethene photosensitizer. J. Med. Chem. 2010, 53, 7337-7343.

28. Boucher, H.W.; Talbot, G.H.; Bradley, J.S.; Edwards, J.E.; Gilbert, D.; Rice, L.B.; Scheld, M.; Spellberg, B.; Bartlett, J. Bad bugs, no drugs: No eskape! An update from the infectious diseases society of america. Clin. Infect. Dis. 2009, 48, 1-12.

29. Hidron, A.I.; Edwards, J.R.; Patel, J.; Horan, T.C.; Sievert, D.M.; Pollock, D.A.; Fridkin, S.K. Nhsn annual update: Antimicrobial-resistant pathogens associated with healthcare-associated infections: Annual summary of data reported to the national healthcare safety network at the centers for disease control and prevention, 2006-2007. Infect. Control Hosp. Epidemiol. 2008, 29, 996-1011.

30. Bartelmess, J.; Weare, W.W. Preparation and characterization of multi-cationic bodipys and their synthetically versatile precursors. Dyes Pigment. 2013, 97, 1-8.

31. Nicolau, D.P. Current challenges in the management of the infected patient. Curr. Opin. Infect. Dis. 2011, 24, S1-S10.

32. Horowitz, B.; Williams, B.; Rywkin, S.; Prince, A.M.; Pascual, D.; Geacintov, N.; Valinsky, J. Inactivation of viruses in blood with aluminum phthalocyanine derivatives. Transfusion 1991, 31, 102-108. 
33. Ben-Hur, E.; Hoeben, R.C.; van Ormondt, H.; Dubbelman, T.M.; van Steveninck, J. Photodynamic inactivation of retroviruses by phthalocyanines: The effects of sulphonation, metal ligand and fluoride. J. Photochem. Photobiol. B 1992, 13, 145-152.

34. Smetana, Z.; Mendelson, E.; Manor, J.; van Lier, J.E.; Ben-Hur, E.; Salzberg, S.; Malik, Z. Photodynamic inactivation of herpes viruses with phthalocyanine derivatives. J. Photochem. Photobiol. B 1994, 22, 37-43.

35. Gaspard, S.; Tempete, C.; Werner, G.H. Studies on photoinactivation by various phthalocyanines of a free or replicating non-enveloped virus. J. Photochem. Photobiol. B 1995, 31, 159-162.

36. Macdonald, I.J.; Dougherty, T.J. Basic principles of photodynamic therapy. J. Porphyr. Phthalocyanines 2001, 5, 105-129.

37. Trias, J.; Benz, R. Permeability of the cell wall of mycobacterium smegmatis. Mol. Microbiol. 1994, 14, 283-290.

38. Padilla, E.; Llobet, E.; Domenech-Sanchez, A.; Martinez-Martinez, L.; Bengoechea, J.A.; Alberti, S. Klebsiella pneumoniae acrab efflux pump contributes to antimicrobial resistance and virulence. Antimicrob. Agents Chemother. 2010, 54, 177-183.

39. Park, B.J.; Wannemuehler, K.A.; Marston, B.J.; Govender, N.; Pappas, P.G.; Chiller, T.M. Estimation of the current global burden of cryptococcal meningitis among persons living with HIV/aids. AIDS 2009, 23, 525-530.

40. McFadden, D.; Zaragoza, O.; Casadevall, A. The capsular dynamics of cryptococcus neoformans. Trends Microbiol. 2006, 14, 497-505.

41. McFadden, D.C.; de Jesus, M.; Casadevall, A. The physical properties of the capsular polysaccharides from cryptococcus neoformans suggest features for capsule construction. J. Biol. Chem. 2006, 281, 1868-1875.

42. Zaragoza, O.; Rodrigues, M.L.; de Jesus, M.; Frases, S.; Dadachova, E.; Casadevall, A. The capsule of the fungal pathogen cryptococcus neoformans. Adv. Appl. Microbiol. 2009, 68, $133-216$.

43. Frases, S.; Pontes, B.; Nimrichter, L.; Rodrigues, M.L.; Viana, N.B.; Casadevall, A. The elastic properties of the cryptococcus neoformans capsule. Biophys. J. 2009, 97, 937-945.

44. Bartelmess, J.; Weare, W.W.; Latortue, N.; Duong, C.; Jones, D.S. Meso-pyridyl bodipys with tunable chemical, optical and electrochemical properties. New J. Chem. 2013, 37, 2663-2668.

45. Bacon, J.; Hatch, K.A. Continuous culture of mycobacteria. In Methods in Molecular Biology, 2nd ed.; Parish, T., Brown, A.C., Eds.; Humana Press: New York, NY, USA, 2009; pp. 153-171.

46. Budhathoki-Uprety, J.; Peng, L.; Melander, C.; Novak, B.M. Synthesis of guanidinium functionalized polycarbodiimides and their antibacterial activities. ACS Macro Lett. 2012, 1, 370-374.

47. Wilkinson, F.; Helman, W.P.; Ross, A.B. Quantum yields of photosensitized formation of the lowest electronically excited singlet state of molecular oxygen in solution. J. Phys. Chem. Ref. Data 1993, 22, 113-262.

48. Rachford, A.A.; Goeb, S.; Castellano, F.N. Accessing the triplet excited state in perylenediimides. J. Am. Chem. Soc. 2008, 130, 2766-2767. 
49. Feese, E.; Sadeghifar, H.; Gracz, H.S.; Argyropoulos, D.S.; Ghiladi, R.A. Photobactericidal porphyrin-cellulose nanocrystals: Synthesis, characterization, and antimicrobial properties. Biomacromolecules 2011, 12, 3528-3539.

Sample Availability: Samples of the compound DIMPy-BODIPY are available from the authors.

(C) 2015 by the authors; licensee MDPI, Basel, Switzerland. This article is an open access article distributed under the terms and conditions of the Creative Commons Attribution license (http://creativecommons.org/licenses/by/4.0/). 